Mosing \& Ullén

Genetic influences on music specialization

\title{
Genetic influences on musical specialization: a twin study on choice of instrument and music genre
}

Miriam A. Mosing ${ }^{1,2}$ and Fredrik Ullén ${ }^{1}$

${ }^{1}$ Department of Neuroscience, Karolinska Institutet, Stockholm, Sweden. ${ }^{2}$ Department of Medical Epidemiology and Biostatistics, Karolinska Institutet, Stockholm, Sweden

Address for correspondence: Miriam A. Mosing, Department of Neuroscience, Karolinska Institute, Retzius y 8, SE-171 77 Stockholm, Sweden. Miriam.Mosing@ki.se

Though severar studies show that genetic factors influence individual differences in musical engagement, aptitude, and achievement, no study to date has investigated whether specialization among musically active individuals in terms of choice of instrument and genre is heritable. Using a large twin cohort, we explored whether individual differences in instrument choice, instrument category, and the type of music individuals engage in can entirely be explained by the environment or are partly due to genetic influences. About 10,000 Swedish twins answered an extensive questionnaire about music-related traits, including information on the instrument and genre they played. Of those, 1259 same-sex twin pairs both reported to either play an instrument or sing. We calculated the odds ratios (ORs) for concordance in music choices (if both twins played) as compared between identical and nonidentical twin pairs, with significant ORs indicating that identical twins are more likely to engage in the same type of music-related behavior compared to nonidentical twins. Results showed that for almost all music-related variables, the odds were significantly higher for identical twins to play the same musical instrument or music genre, suggesting significant genetic influences on such music specialization. Possible interpretations and implications of the findings are discussed.

Keywords: twin; music-related choice; concordance; instrument; gene-environment

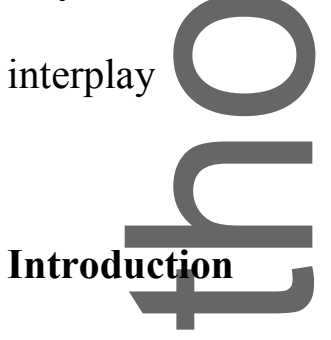

Human music is a highly diverse phenomenon. Different types of music show characteristic differences in many parameters, such as which instruments are used; scales and tuning systems; melodic, harmonic, and rhythmic conventions; the formal structure of musical

This is the author manuscript accepted for publication and has undergone full peer review but has not been through the copyediting, typesetting, pagination and proofreading process, which may lead to differences between this version and the Version of Record. Please cite this article as doi:

$\underline{10.1111 / \text { nyas. } 13626 .}$.

This article is protected by copyright. All rights reserved. 
pieces; the use of notation and improvisation; and many other features. ${ }^{1}$ The impressive cross-cultural variation in the musics of the world is studied within the field of ethnomusicology. ${ }^{2}$ Even within contemporary Western societies, there is considerable variation among musically active people, for example, with regard to choice of instrument, genres and musical styles, and the social context of music making. In general, such variation in cultural engagement correlates substantially with social and cultural background, ${ }^{3-5}$ and differences in eeonomic and cultural capital between social groups have often been suggested to be a fundamental cause of observed differences in cultural practices and preferences within a society (see, e.g., Refs. 5 and 6).

However, research using genetically informative designs shows that individual differences and familial similarity in cultural traits can be substantially influenced by genetic factors. In one early study, Martin and coworkers, ${ }^{7}$ for example, analyzed family resemblance in social attitudes, finding that when assortative mating was taken into account, there was strong support for a genetic model, with surprisingly small evidence for vertical cultural transmission from parents to children. Numerous other studies have demonstrated genetic influences on culturally relevant traits such as political orientation, ${ }^{8}$ religiosity, ${ }^{9}$ vocational and recreationalinterests, ${ }^{10}$ moral thinking, ${ }^{11}$ parenting behavior and family processes, ${ }^{12}$ and social values. ${ }^{13}$ Several theoretical models have also been proposed to account for the often complex interactions between genetic and nongenetic factors in cultural evolution (for a recent review, see Ref. 14).

Studies from our group and other laboratories have found evidence for substantial genetic influences on various music-related traits (for a review, see Ref. 15), including musical aptitude, ${ }^{10-18}$ creative achievement in music, ${ }^{19}$ music training, ${ }^{16}$ and enjoyment of music, operationalized as the frequency of psychological flow experiences during music making. ${ }^{20}$ Notably, all these studies analyze general outcomes relating to musical ability and 
engagement, without taking into account the large variation in forms of musical activity discussed above. Specifically, to our knowledge, it has not previously been investigated whether what could be considered subcultural specialization among musically active people-that is, choice of genre and musical instrument - is also influenced by genetic factors. Here, we investigate this question in a large cohort of Swedish twins, by testing whether MZ twins show a significantly higher concordance for choice of main instrument and genre than DZ twins. Instrument choice was considered by looking at concordance for specific instruments as well as for broader instrument categories which differ in basic playing techniques and modes of sound production. The decision which main instrument to play is often made early in life, and can be influenced by the playing individual as well as other people such as parents and teachers. In a final exploratory analysis, we investigated concordance patterns for instrument choice separately for twin pairs who reported to have chosen their instrument themselves, and twin pairs who reported that other people were the main influence on their instrument selection.

\section{Methods \\ Participants and data collection}

Data for the present study were collected in 2012 and 2013 as part of a web survey administered to an adult cohort of twins registered with the Swedish Twin Registry. ${ }^{21,22}$ The web survey was designed to collect extensive information on both music-related variables and general psychological traits and the study was approved by the Regional Ethics Review Board in Stockholm (Dnr 2011/570-31/5). As part of the web survey, all participants gave informed consent before continuing with the survey. In total, 11,543 twins aged between 27 and 54 participated in the survey, although not all twins responded to each question. Zygosity was determined based on a questionnaire about intrapair resemblance. In the Swedish Twin 
Registry, agreement on zygosity determination based on this questionnaire and DNA genotyping is more than $98 \% .^{21,22}$ (For further information on the data collection procedure and the web survey, see Ref. 16.) Of the participants, 7786 (67\%) reported to play or ever have played an instrument (including singing) and reported their main instrument. Here, we were interested in within-pair concordance in music-related choices (i.e., instrument of choice and music genre); therefore, only pairs where both twins indicated to play an instrument (or sing) were included here - in total 1685 pairs. Of those, 56 pairs had missing zygosity and were excluded from the analyses.

\section{Measures}

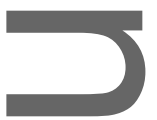

Instrument choiee. With an open question, participants were asked to freely indicate which main instrument they played. Note that they were asked to report only one main instrument. Participants reported to play among 45 different instruments (including song, choir, and whistling) - for detailed list of the different instruments reported in the full sample and numbers per instrument, see Supplementary Table S1 (online only). Based on their response, twin pairs were then coded as playing the same or different instruments (concordant versus discordant for instrument choice).

Instrument type. The above response (instrument choice) was then classified into the following instrument types (see Supplementary Table S1, online only, for details): bowed and plucked (1), voice (2), keyboard (3), woodwind (4), brass (5), and percussion (6). Note that three individuals reported to play a sequencer. As this did not fit any of the above types, their score was set to missing for the instrument type variable. Again, based on the instrument type, twin pairs were coded as playing the same or different instrument type (concordant versus discordant for instrument type). 
Influence on instrument choice. Participants who responded that they play or had played an instrument were also asked who the main influence on their instrument choice was. Response alternatives were "myself," "parent or other relative," "friend," "music teacher," and "other person." To analyze whether twin concordance patterns differed between participants who reported that they chose their instrument themselves and those who did not, responses on this item were dichotomized into self-chosen (“myself") and not self-chosen (all other response categories).

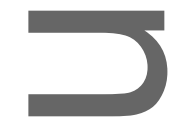

Music genres. Participants who indicated that they (had) played an instrument were asked "Which of the following types of music did you perform or practice?" with the following response options (several could be selected): "classical Western art music," "modern Western art music," "jazz music," "pop/rock," "folk and world music," and "other types of music." The question was asked for different periods in life, that is, when participants were $0-5$ years, 6-11 years, 12-17 years, and 18 years old until presence. From this, three variables for music genre were derived. Art music genre-individuals who had indicated that at any stage in their lives they played any of the two types of Western art music were coded as one on this variable and the remainder was coded as zero. Jazz genre — individuals who had indicated that at any stage in their lives they have played Jazz music were coded as one on this variable and the remainder was coded as zero. Last, Modern genre-with individuals coded as one if they played pop/rock or folk/world music at any stage in their lives.

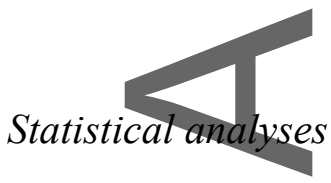

Preliminary analyses. Preliminary analyses were conducted to test for any potential differences in means or numbers in the various variables (including age and sex) between the 
two zygosity groups. This was done using logistic or linear regression with zygosity type (MZ/DZ) as the independent variable and the various instrument types and genres (coded as dummy variables), sex and age (continuous) as dependent variables. To correct for correlation within twin-pairs, the robust standard error estimator for clustered observations was used.

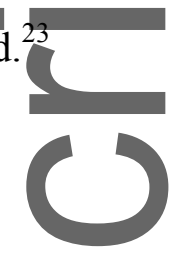

Twin analyses. MZ twins share all their genes, whereas DZ twins on average share $50 \%$ of their segregating genes. Therefore, assuming equal environments (for MZ versus DZ twins on average), if individual differences in a trait (e.g., instrument choice) were entirely due to genetic influences, we would expect a twin pair correlation of 1 for MZ pairs, that is, the two twins of an MZ pair would always chose to play the same instrument. DZ twins in this scenario would show a twin correlation of 0.5 (resemble each other half the time), as they only share half of their genetic makeup. On the other hand, if environmental factors were the only source of variance in a trait, we would expect the twin pair correlation not to differ between $\mathrm{MZ}$ and DZ twin pairs (though correlations could range anywhere between 0 and 1 depending on whether the environmental impact is shared between the twins or not), that is, if the environment was the sole determinant of instrument choice and as twin pairs regardless of $\mathrm{MZ}$ or DZ are expected to be exposed to similar environments on average, then we would expect no difference in within-pair resemblance in instrument choice between $\mathrm{MZ}$ and DZ pairs. Hence, environmental versus genetic influences predict different patterns of MZ versus DZ twin pair resemblance.

Here, we explored rates of concordance versus discordance for music-related choices in music playing twins, comparing the odds for music playing MZ twins to choose the same instrument/genre with the odds for DZ twins, resulting in an odds ratio (OR). Logistic 
regression was conducted with zygosity status (MZ versus same-sex DZ) as the independent variable and concordance/discordance of pairs for each respective music-related outcome as dependent variable. A significant $\mathrm{OR}$ indicates that $\mathrm{MZ}$ twins are significantly more likely to resemble each other in their music-related choices compared to DZ twins, which in turn would suggest that genetics play a significant role in explaining individual differences in music-related choices. Similarly, if MZ and DZ twins do not differ significantly in their resemblance, that is, nonsignificant ORs, only environmental influences were of relevance. To control for potential sex influences on within-pair resemblance, only same-sex DZ pairs were included in the analyses. ORs were calculated for within-pair resemblance on instrument choice, instrument type, and the three instrument genres. In addition, to confirm findings on instrument choice, also differences in within-pair resemblance between MZ and DZ twins in the following specific instruments were calculated: piano, guitar, and singing (the most frequently indicated music "instruments" in the present sample).

In a final analysis, we investigated whether a higher MZ than DZ concordance for instrument choice would be seen regardless of who chose the instrument, that is, the twin him/herself or someone else chose for the twin. This was done to test whether the increased concordance rate in MZ twins could be explained by external influences outside of the twins themselves (i.e., family members) who are more likely to choose the same instrument for MZ than DZ twins or whether this finding is due to a more innate urge (or both). To investigate this issue, we repeated the analyses separately for twin pairs where both twins had chosen their instrument themselves, and twin pairs where both twins reported that someone else chose the instrument. All analyses were conducted in Stata 14 (Ref. 23). 


\section{Results}

After excluding opposite-sex twin pairs $\left(\mathrm{N}_{\text {pairs }}=370\right)$ to avoid potential confounding by sex differences, the final sample consisted of 1259 same-sex twin pairs-803 monozygotic (MZ) and 456 same-sex dizygotic (DZ) pairs. Preliminary analyses indicated that there were no significant-differences in the variables of interest between the zygosity groups except for age, with MZ being slightly younger than DZ twins (Table 1). Therefore, although it is unlikely that age would have an impact on the within-pair concordance rates, all analyses below were corrected for age.

Results of the statistical analyses of MZ and DZ concordance rates are summarized in Table 2. Concordant rates for instrument choice and type were relatively high (around 40$50 \%$ ), though somewhat lower for the three most common instruments. For the three genres, concordance was even higher (up to $86 \%$ ) partly due to the fact that twins could choose more than one genre and in most cases also chose several. Without exception, concordance rates between MZ pairs were higher than for DZ twins. OR analysis showed the difference in MZ/DZ concordance to be highly significant $(P<0.001)$ for both instrument choice and instrument type. When further exploring differences in concordance rates between zygosities for the three most common instruments, differences were highly significant for piano and song, while the trend for higher MZ concordance did not reach significance for guitar, most likely due to the small sample size for this instrument (Table 2). ORs for the music genres (art music, jazz, and modern/folk) were somewhat lower but still in all cases significant at $P$ $<0.05$.

Some of the twins reported that they chose their main instrument themselves $(\mathrm{N}=348)$, while others responded that some other person, such as a parent or teacher, was the main influence on their instrument choice $(\mathrm{N}=390)$. In a final analysis, we investigated whether a higher MZ than DZ concordance for instrument choice would be seen regardless of who 
made the choice. Analyses were repeated separately for twin pairs where both twins had chosen their instrument themselves, and twin pairs where both twins reported that someone else chose the instrument. As summarized in Table 3, MZ twins were significantly more concordant than DZ twins for instrument choice and instrument type, regardless of whether they chose the instrument themselves or not. ORs for pairs where both twins reported to have chosen theirinstrument themselves were slightly higher than for those where others chose the instrument, though confidence intervals were largely overlapping. Interestingly, concordance rates were somewhat higher in both MZ and especially DZ twins in those pairs reporting that others chose thein instrument for them.

\section{Discussion}

We investigated whether specialization, that is, choice of instrument and genre, among musically active Swedish twins is at least partly genetically influenced. Analyses of withinpair similarity (concordance) showed that musically active $\mathrm{MZ}$ twin pairs were more concordant than DZ pairs for choice of musical genre as well as instrument. The latter effect was seen for both instrument categories and specific instruments. The findings give a strong indication that genes indeed influence these aspects of specialization among musically active individuals, and are in line with the broader literature showing genetic influences on individual variability in other music-related traits (for detailed reviews, see, e.g., Refs. 24-26) and cultural variables such as political and religious orientation, ${ }^{8,9}$ interests $^{27}$ and hobbies, ${ }^{10}$ as well as other "environmental" choices. ${ }^{28}$ The differences in twin concordance were robust across different instrument types and genres. Notably, for instance, genetic influences were indicated for both piano playing and singing, although the decision to play the piano seems likely to be influenced by whether a piano is present in the shared environment of the twins, 
while — in contrast — singing requires no extra equipment and is in principle accessible to everyone.

In general, two mechanisms for the observed findings appear plausible. The first is active gene-environment correlation suggesting the musical twins actively seek out an environment (i.e., preferences and decisions regarding musical specialization) based on their innate traits. One psychological modality, which is likely to mediate such associations, is personality. Extensive, pioneering work on the personality profile of musicians was performed by Kemp, ${ }^{29}$ who documented correlations between personality and musical instrument, as well as other specializations within the musical profession (performers, composers, teachers, etc.). Later studies have also used various personality measures to document associations between both instrument and genre choice and personality facets among musicians (see, e.g., Refs. 30-33). Musical specialization may also be related to cognitive abilities. Benedek and coworkers thus report that jazz musicians score higher than classical musicians on test of divergent thinking. ${ }^{33}$ Other studies have, for example, found percussionists to outperform nonpercussionist musicians on timing tasks. ${ }^{34}$ This suggests that differences in the relative strengths of different musical aptitudes (melody, rhythm, etc.) may have an impact on instrument choice. Finally, it is likely that the decision to play a musical instrument also is influenced by relevant physical traits, such as hand size for pianists and voice quality for singers (for a discussion, see Ref. 35). In summary, these observations suggest that genetic effects on instrument and genre choice, at least in part, could be explained by differences in personality, cognitive abilities, and relevant physical traits. A hypothesis to test in future studies is therefore that instrument choice overlaps genetically with such traits. Notably, genetic correlations with personality have been found for other cultural traits, such as political orientation. ${ }^{8}$ 
A second general mechanism that could underlie the observed concordance patterns is reactive gene-environment correlation. ${ }^{36}$ The (genetically mediated) behavior and traits of the twins elicit particular reactions (e.g., in social interactions relating to their musical activities) from other people, such as parents, peers, and music teachers, and to the extent that $\mathrm{MZ}$ pairs resemble each other more on traits potentially related to music than the $\mathrm{DZ}$ pairs (due to genetic influences), it is likely that they will evoke more similar behaviors from parents in terms of support and advice regarding questions about musical specialization.

The fact that higher MZ than DZ concordances for instrument choice were seen for twin pairs regardless of whether they reported to have chosen their instrument themselves or that other people were influential in the choice could be seen as an indication that the underlying mechanisms are complex, and may involve behavioral tendencies of the twin themselves as well as reactions they evoke from people in their surroundings (i.e., active and reactive geneenvironment correlations). The trend for higher odds for concordance in MZ compared to DZ in those pairs indicating that they chose the instrument themselves could suggest that the net relative influence of genetic factors was somewhat higher in that group. This is not unexpected as less interference from other people would likely result in a lower environmental contribution to the variance in instrument choice and, consequently, a higher heritability. This is in line with the higher concordance rates in both $\mathrm{MZ}$ and DZ twins in those pairs where others chose the instrument for them, suggesting that others are more likely to simplychoose the same instrument for both twins of a pair.

Unfortunately, with the present data, we cannot fully rule out a violation of the equal environment assumption (EEA), that is, that $\mathrm{MZ}$ twins are more correlated in their exposure to environmental events of importance for the trait of interest simply due to the environment treating MZ twins more similar because of the knowledge of dealing with identical rather than fraternal twins. However, the fact that we observed a higher concordance rate in $\mathrm{MZ}$ 
twins also in pairs where both twins actively chose their instrument suggests that the findings are unlikely explained by this type of violation, but we cannot dismiss the possibility that the individual decisions are potentially influenced by other environmental factors which make MZ more similar. To date, the majority of research testing the EEA as shown that it holds (e.g., Refs. 37 and 38). In addition, it is important to keep in mind that the present data are based on web-based self-report, which could be subject to bias. However, it appears unlikely that this would have a large effect on the variables of main interest - choice of instrument and music genre. Also, random measurement errors resulting from incorrect memories of which instrument or genre the participant had engaged in should, if anything, tend to decrease twin concordances.

In conctusion, the present findings indicate that even expertise-related outcomes which generally have been regarded as environmental, such as music specialization, are partly genetically mediated. In line with the recent developments in the field of expertise research, the present findings highlight the importance of a multifactorial understanding of expertise, which takes into account the interplay between an individual's genetic predispositions with environmental factors to create expertise-related behavior (for a detailed discussion, see Ref. 26).

A final note is that the present findings could be of potential interest for the interpretation of imaging studies of the neural correlates of musical specialization. For instance, earlier studies have found differences between piano and string players in the anatomy of the primary motor cortices and the pyramidal tracts, ${ }^{39,40}$ and between singers and instrumentalist in the arcuate fasciculus. ${ }^{41}$ It certainly appears plausible that such highly specific instrumentrelated differences in neuroanatomy between groups of musicians are the consequences of experience and plasticity. Furthermore, recent studies using a cotwin control design to control for genetic factors provide novel support for that musical training indeed can have causal 
effects on regional neuroanatomy. ${ }^{42}$ However, the results of the present study suggest that potential genetic confounding has to be kept in mind even when considering highly specific differences between specialists in the same domain of expertise. Apparently, genetic influences-on music-related traits are not limited to global effects on aptitude, motivation, and training, but may even influence the specific directions that musical engagement takes among musically active people.

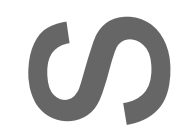

\section{Acknowledgments}

The present work was supported by the Bank of Sweden Tercentenary Foundation (M110451:1). We thank the twins for their participation.

\section{Competing interests}

The authors declare no competing interests.

\section{Reference}

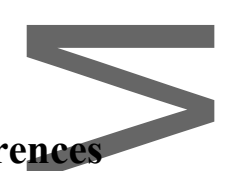

1. Sloboda, J.A. 1999. The musical mind in context: culture and biology. In The Musical Mind. The Cognitive Psychology of Music.: 239-268. Oxford, UK: Oxford University Press. 2. Nettl, B. \& T. Rommen. 2017. Excursions in World Music. New York: Routledge. 3. Eurostat, 2011. Cultural Statistics. Luxembourg: Publications Office of the European Union.

4. Swedish Art Council. 2008. New Cultural Habits. Cultural Habits in Sweden in a 30 Years Perspective: 1976-2006. Sweden: Elanders.

5. Veenstra, G. 2015. Class position and musical tastes: a sing-off between the cultural omnivorism and Bourdieusian homology frameworks. Can. Rev. Sociol. 52: 134-159.

6. Bourdieu, P. 1984. Distinction: A Social Critique of the Judgement of Taste. Cambridge, MA: Harvard University Press.

7. Martin, N.G., L.J. Eaves, A.C. Heath, et al. 1986. Transmission of social attitudes. Proc. Natl. Acad.Sci. USA 83: 4364-4368.

8. Kandler, C., W. Bleidorn \& R. Riemann. 2012. Left or right? Sources of political orientation: the roles of genetic factors, cultural transmission, assortative mating, and personality. J. Pers. Soc. Psychol. 102: 633-645. 
9. Kandler, C. \& R. Riemann. 2013. Genetic and environmental sources of individual religiousness: the roles of individual personality traits and perceived environmental religiousness. Behav. Genet. 43: 297-313.

10. Lykken, D.T., T.J. Bouchard, M. McGue, et al. 1993. Heritability of interests---a twin study. J. Appl. Rsychol. 78: 649-661.

11. Campbell,J., J.A. Schermer, V.C. Villani, et al. 2009. A behavioral genetic study of the dark triad of personality and moral development. Twin Res. Hum. Genet. 12: 132-136. 12. Moore, G.A. \& J.M. Neiderhiser. 2014. Behavioral genetic approaches and family theory. J. Fam. Theory Rev. 6: 18-30.

13. Polderman, T.J.C., B. Benyamin, C.A. de Leeuw, et al. 2015. Meta-analysis of the heritability of human traits based on fifty years of twin studies. Nat. Genet. 47: 702-709.

14. Creanza, N., O. Kolodny \& M.W. Feldman. 2017. Cultural evolutionary theory: how culture evolves and why it matters. Proc. Natl. Acad. Sci. USA. DOI:

10.1073/pnas.1620732114.

15. Mosing, M.A. \& F. Ullén. 2016. Genetic influences on musical giftedness, talent and practice. In Musical Prodigies: Interpretations from Psychology, Music Education,

Musicology and Ethnomusicology. G.E. McPherson, Ed.: 156-167. Oxford, UK: Oxford University Press.

16. Mosing, M.A., G. Madison, N.L. Pedersen, et al. 2014. Practice does not make perfect: no causal effect of music practice on music ability. Psychol. Sci. 25: 1795-1803.

17. Drayna,D., A. Manichaikul, M. de Lange, et al. 2001. Genetic correlates of musical pitch reeognition in humans. Science 291: 1969-1972.

18. Mosing, M.A., K.J.H. Verweij, G. Madison, et al. 2016. The genetic architecture of correlations between perceptual timing, motor timing, and intelligence. Intelligence 57: 3340.

19. Mosing, M.A., K.J.H. Verweij, G. Madison, et al. 2015. Did sexual selection shape human music? Testing predictions from the sexual selection hypothesis of music evolution using a large genetically informative sample of over 10,000 twins. Evol. Hum. Behav. 36: 359-366.

20. Butkovié, A., F. Ullén \& M.A. Mosing. 2015. Personality and related traits as predictors of music practice: underlying environmental and genetic influences. Personal Individ. Differ. 74: 133-138.

21. Lichtenstein, P., P.F. Sullivan, S. Cnattingius, et al. 2006. The Swedish Twin Registry in the third millennium: an update. Twin Res. Hum. Genet. 9: 875-882.

22. Lichtenstein, P., U. De Faire, B. Floderus, et al. 2002. The Swedish Twin Registry: a unique resource for clinical, epidemiological and genetic studies. J. Intern. Med. 252: 184205.

23. StataCorp. 2016. Stata: release 14.2. In Statistical Software. College Station, TX: StataCorp LP.

24. Mosing, M.A., I. Peretz \& F. Ullén. 2017. Genetic influences on musical expertise. In The Science of Expertise: Behavioral, Neural, and Genetic Approaches to Complex Skill. D.Z. Hambrick, G. Campitelli \& B.N. Macnamara, Eds. New York: Routledge Pychology Press. 
25. Tan, Y.T., G.E. McPherson, I. Peretz, et al. 2014. The genetic basis of music ability. Front. Psychol. 5: 658.

26. Ullen, F., D.Z. Hambrick \& M.A. Mosing. 2016. Rethinking expertise: a multifactorial gene-environment interaction model of expert performance. Psychol. Bull. 142: 427-446.

27. Bouchard,T.J., Jr. \& M. McGue. 2003. Genetic and environmental influences on human psychological differences. J. Neurobiol. 54: 4-45.

28. Vinkhuyzen, A.A., S. van der Sluis, E.J. de Geus, et al. 2010. Genetic influences on 'environmental' factors. Genes Brain Behav. 9: 276-287.

29. Kemp, A.E. 1996. The Musical Temperament. Psychology and Personality of Musicians. Oxford, UK: Oxford Univ Press.

30. Buttsworth, L.M. \& G.A. Smith. 1995. Personality of Australian performing musicians by gender and by instrument. Personal Individ. Differ. 18: 595-603.

31. MacLellan, C.R. 2011. Differences in Myers--Briggs personality types among high school band, orchestra, and choir members. J. Res. Music Educ. 59: 85-100.

32. Cameron, J.E., M. Duffy \& B. Glenwright. 2015. Singers take center stage!

Personality traits and stereotypes of popular musicians. Psychol. Music 43: 818-830.

33. Benedek, M., B. Borovnjak, A.C. Neubauer, et al. 2014. Creativity and personality in classical, jazz and folk musicians. Personal Individ. Differ. 63: 117-121.

34. Cicchini, M., R. Arrighi, L. Cecchetti, et al. 2012. Optimal encoding of interval timing in expert percussionists. J. Neurosci. 32: 1056-1060.

36. Plomin, R., J.C. DeFries, V.S. Knopik, et al. 2013. Behavioral Genetics. New York:

Worth Publishers.

37. Kendler, K.S., M.C. Neale, R.C. Kessler, et al. 1993. A test of the equal-environment assumption in twin studies of psychiatric illness. Behav. Genet. 23: 21-27.

38. Derks, E.M., C.V. Dolan \& D.I. Boomsma. 2006. A test of the equal environment assumption (EEA) in multivariate twin studies. Twin Res. Hum. Genet. 9: 403-411.

39. Bangert, M. \& G. Schlaug. 2006. Specialization of the specialized in features of external human brain morphology. Eur. J. Neurosci. 24: 1832-1834.

40. Rüber, T. R. Lindenberg \& G. Schlaug. 2015. Differential adaptation of descending motor tracts in musicians. Cereb. Cortex 25: 1490-1498.

41. Halwani, G.F., P. Loui, T. Rüber, et al. 2011. Effects of practice and experience on the arcuate fasciculus: comparing singers, instrumentalists, and non-musicians. Front. Psychot. 2:156.

42. de Manzano, Ö. \& F. Ullén. 2018. Same genes, different brains: neuroanatomical differences between monozygotic twins discordant for musical training. Cereb. Cortex 28: 387-394.

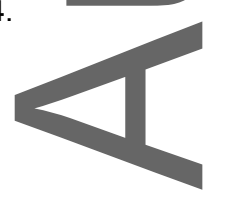


Table 1. Descriptive statistics on the final sample showing the mean levels (SD) and counts (\%) for the various variables in the full sample, in $\mathrm{MZ}$ and DZ twins, as well as testing for significant differences between MZ and DZ twins

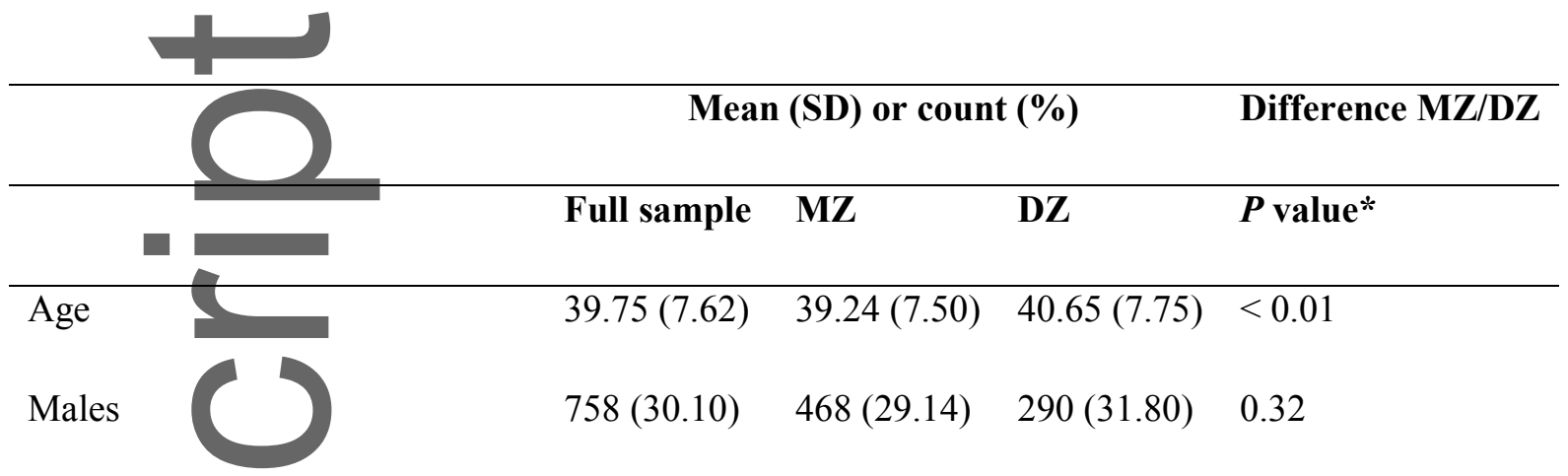

Instrument type (Categories 1-6)

(1) Bowed/plucked $573(22.77) \quad 372(23.16) \quad 201(22.04) \quad 0.57$

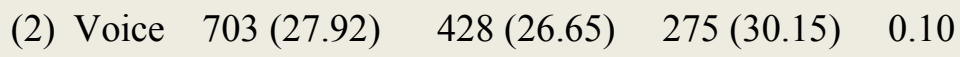
(3) Keyboard $551(21.88) \quad 352(21.92) \quad 199(21.82) \quad 0.96$
(4) Woodwind $535(21.25) \quad 360(22.42) \quad 175(19.19) \quad 0.10$
$\begin{array}{llll}\text { (5) Brass } 94(3.73) \quad 55(3.42) \quad 39(4.28) & 0.35\end{array}$

(6) Percussion $62(2.46) \quad 39(2.43) \quad 23(2.52) \quad 0.89$

Instrument (3 most common)

$\begin{array}{llllll}\text { Piano } & 499(19.82) & 318(19.80) & 181(19.85) & 0.98 \\ \text { Guitar } & 344(13.66) & 228(14.20) & 116(12.72) & 0.36 \\ & \text { Song } & 690(27.40) & 419(26.09) & 271(29.71) & 0.09\end{array}$

Genre

$$
\begin{array}{rcccc}
\text { Art music } & 739(45.06) & 461(43.37) & 278(48.18) & 0.09 \\
\text { Jazz } & 222(13.54) & 143(13.45) & 79(13.69) & 0.91 \\
& & & & \\
\text { Modern/folk } & 1490(90.85) & 976(91.82) & 514(89.08) & 0.09
\end{array}
$$

*P values are corrected for relatedness of the sample. 
Table 2. Twin concordances

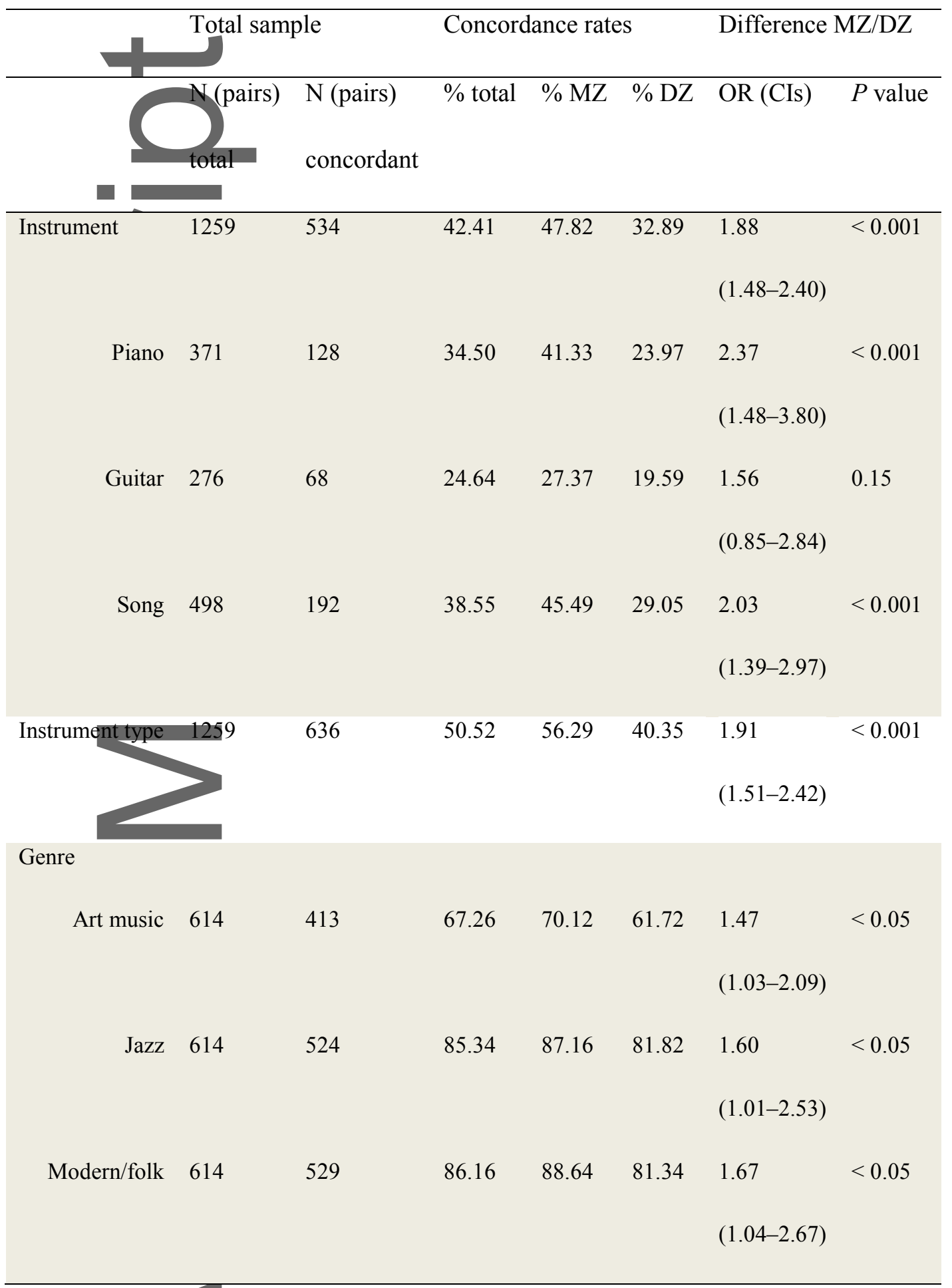

NoTE: Only 614 twin pairs both filled out the genre questions and that more than one genre could be chosen. For each variable, the columns show the total number of twin pairs and number of concordant twin pairs; concordance rates (\%) for the total sample, and separately for MZ and DZ twin pairs; and odds ratios (OR) for the MZ/DZ difference with $95 \%$ confidence intervals (CIs) in parentheses and $P$ values. For instrument choice (instrument), separate statistics are also provided for the three most common individual instruments (piano, guitar, and song).

This article is protected by copyright. All rights reserved. 
Table 3. Additional analyses comparing concordance rates in $\mathrm{MZ}$ and $\mathrm{DZ}$ twins in the instrument variables in twin pairs where both indicated to have chosen their instrument themselves versus those where others influenced their instrument choice

\begin{tabular}{|c|c|c|c|c|c|c|}
\hline \multirow{2}{*}{$\begin{array}{l}\text { Pairs where instrument for } \\
\text { both twins was ehosen by: }\end{array}$} & & & \multicolumn{2}{|c|}{$\begin{array}{l}\text { Concordance } \\
\text { rates }\end{array}$} & \multicolumn{2}{|c|}{ Dif. MZ/DZ } \\
\hline & Variable & $\begin{array}{l}\mathrm{N} \\
\text { (pairs) }\end{array}$ & $\% \mathrm{MZ}$ & $\% \mathrm{DZ}$ & $\begin{array}{l}\text { OR } \\
\text { (CIs) }\end{array}$ & $\begin{array}{l}P \\
\text { value }\end{array}$ \\
\hline \multirow[t]{4}{*}{ Themselves } & Instrument & 348 & 44.20 & 21.77 & 2.92 & $<$ \\
\hline & & & & & $\begin{array}{l}(1.76- \\
4.85)\end{array}$ & 0.001 \\
\hline & Instrument & 348 & 54.91 & 28.23 & 3.15 & $<$ \\
\hline & type & & & & $\begin{array}{l}(1.96- \\
5.06)\end{array}$ & 0.001 \\
\hline \multirow[t]{2}{*}{ Others } & Instrument & 390 & 64.94 & 45.38 & $\begin{array}{l}2.08 \\
(1.33- \\
3.25)\end{array}$ & 0.001 \\
\hline & $\begin{array}{l}\text { Instrument } \\
\text { type }\end{array}$ & 390 & 70.85 & 52.94 & $\begin{array}{l}2.00 \\
(1.27- \\
3.15)\end{array}$ & 0.003 \\
\hline
\end{tabular}




\section{University Library}

\section{- M M N E R VA A gateway to Melbourne's research publications}

Minerva Access is the Institutional Repository of The University of Melbourne

Author/s:

Mosing, MA;Ullen, F

Title:

Genetic influences on musical specialization: a twin study on choice of instrument and music genre

Date:

2018-07-01

Citation:

Mosing, M. A. \& Ullen, F. (2018). Genetic influences on musical specialization: a twin study on choice of instrument and music genre. ANNALS OF THE NEW YORK ACADEMY OF SCIENCES, 1423 (1), pp.427-434. https://doi.org/10.1111/nyas.13626.

Persistent Link:

http://hdl.handle.net/11343/261118 